\title{
Human milk metagenome: a functional capacity analysis
}

Tonya L Ward' ${ }^{1}$, Sergey Hosid ${ }^{1,2}$, Ilya loshikhes ${ }^{1,2^{*}}$ and Illimar Altosaar ${ }^{1,2^{*}}$

\begin{abstract}
Background: Human milk contains a diverse population of bacteria that likely influences colonization of the infant gastrointestinal tract. Recent studies, however, have been limited to characterization of this microbial community by $16 \mathrm{~S}$ rRNA analysis. In the present study, a metagenomic approach using Illumina sequencing of a pooled milk sample (ten donors) was employed to determine the genera of bacteria and the types of bacterial open reading frames in human milk that may influence bacterial establishment and stability in this primal food matrix. The human milk metagenome was also compared to that of breast-fed and formula-fed infants' feces ( $n=5$, each) and mothers' feces $(n=3)$ at the phylum level and at a functional level using open reading frame abundance. Additionally, immune-modulatory bacterial-DNA motifs were also searched for within human milk.

Results: The bacterial community in human milk contained over 360 prokaryotic genera, with sequences aligning predominantly to the phyla of Proteobacteria (65\%) and Firmicutes (34\%), and the genera of Pseudomonas (61.1\%), Staphylococcus (33.4\%) and Streptococcus (0.5\%). From assembled human milk-derived contigs, 30,128 open reading frames were annotated and assigned to functional categories. When compared to the metagenome of infants' and mothers' feces, the human milk metagenome was less diverse at the phylum level, and contained more open reading frames associated with nitrogen metabolism, membrane transport and stress response $(P<0.05)$. The human milk metagenome also contained a similar occurrence of immune-modulatory DNA motifs to that of infants' and mothers' fecal metagenomes.

Conclusions: Our results further expand the complexity of the human milk metagenome and enforce the benefits of human milk ingestion on the microbial colonization of the infant gut and immunity. Discovery of immunemodulatory motifs in the metagenome of human milk indicates more exhaustive analyses of the functionality of the human milk metagenome are warranted.
\end{abstract}

Keywords: Human milk, Microbiome, Metagenome, Bacteria, Illumina, DNA, Open reading frames, Immune-modulatory motifs, Infant feces

\section{Background}

The benefits of human milk compared to the use of commercial infant formulas are largely realized because of its bioactive components, including prebiotics, immune proteins and the microbiome of human milk itself. Breastfeeding is associated with a decreased incidence of gastrointestinal (GI) tract infections [1,2], which is corroborated by several studies that have correlated breastfeeding with a lower incidence of necrotizing

\footnotetext{
*Correspondence: altosaar@uottawa.ca; iioschik@uottawa.ca

'Department of Biochemistry, Microbiology and Immunology; and Ottawa Institute of Computational Biology and Bioinformatics, University of Ottawa, Ottawa, ON, K1H 8M5, Canada

${ }^{2}$ Ottawa Institute of Systems Biology, University of Ottawa, Ottawa, ON, K1H 8M5, Canada
}

enterocolitis in humans and animal models [3-5]. Breastfeeding is also associated with an altered fecal microbiome; two studies showed at two weeks of age over $90 \%$ of the total fecal bacteria of a breast-fed (BF) infant is Bifidobacteria, whereas in most formula-fed (FF) infants Bifidobacteria is non-detectable [6,7]. Because the community of gut-colonizing bacteria prevents adhesion and colonization of pathogenic bacteria whilst stimulating mucosal cell proliferation and enhancing immune development, the types of predominant bacteria in the fecal microbiome of the developing infant can affect the health outcomes of the individual, as has been discussed in a recent review article [8]. Human milk, the infant's first food, is a primary source of ingested 
microbiota. Therefore, it is paramount to fully understand the human milk microbiome and how it might influence colonization of the infant GI tract.

Ingestion of viable bacteria in human milk may lead to effective colonization of the infant GI tract, but the presence of bacterial DNA alone may also hold responsibility for proper infant immune development. For example, unmethylated cytosine phosphate guanine (CpG) dinucleotides within bacterial DNA are known as potent immune stimulators, acting through toll-like receptor 9 [9]. Conversely, immune suppressive motifs including polyguanosine or guanosine cytosine-rich sequences, such as those on the telomere region of mammalian DNA, that can block immune activation induced by CpGs [10]. Recently, immune suppressive motifs (TTAGGG and TCAAGCTTGA) that are able to counter the effects of CpGs have been discovered in Lactobacillus [11]. If immune-modulatory motifs occur in human milk derived DNA, they could contribute to proper immune development by decreasing exaggerated inflammatory responses to colonizing bacteria, which are seen in infants with necrotizing enterocolitis [12].

Human milk bacteria have previously been analyzed by culture-dependent and -independent mechanisms, confirming the presence of a magnitude of bacterial phylotypes [13-20]. In one study, Staphylococcus and Streptococcus dominated the milk microbiome of most mothers, whereas commercially well known bovine milkassociated genera, Lactobacillus and Bifidobacterium, contributed as minor milk microbiota members (2-3\% of genera) [17]. Another study showed that the human milk microbiome changes over time, and may be dependent on the mother's weight and the baby's mode of delivery [20]. Most recent methods for determining the milk microbiome have included amplification of $16 \mathrm{~S}$ ribosomal RNA genes (rRNA) followed by pyrosequencing $[17,20]$. Although this technique is widely accepted as a means to determine microbial diversity, it does present limitations such as a lack of information on the functional capacity of the microbes within the milk matrix and also prevents data accumulation on the types of DNA motifs to which an infant is exposed.

In this study we performed a metagenomic analysis of the bacteria in human milk using Illumina sequencing and the MG-RAST pipeline [21]. The aims were to determine the genera of bacteria in human milk, search for immune-modulatory DNA motifs, and determine the types of bacterial open reading frames (ORFs) in human milk that may influence bacterial presence and stability in this complex yet foundational food matrix.

\section{Results}

\section{Phyla and genera within human milk}

Metagenome sequencing of a pooled human milk sample resulted in 261,532,204 sequenced reads of 51 bp, which were binned into those aligning to the human genome $(186,010,988,72.01 \pm 3.06 \%)$, known prokaryotic genomes $(1,331,996,0.53 \pm 0.16 \%)$ or those not aligning to either category $(74,189,220,27.46 \pm 3.72 \%$, Additional file 1). Using a best hit analysis of the 1,331,996 51-bp sequences, $75 \%$ aligned to Staphylococcus, $15 \%$ to Pseudomonas, 2\% to Edwardsiella, and 1\% to Pantoea, Treponema, Streptococcus and Campylobacter, respectively (Figure 1). The remaining 3\% of the known prokaryotic sequences mapped to 361 bacterial genera, demonstrating the diversity of the human milk metagenome while confirming the presence of key genera like Akkermansia (Additional file 2).

Sequences not aligning to prokaryotic or human genomes with $\mathrm{a} \leq 2 \mathrm{bp}$ mismatch were re-aligned to the human genome with decreased stringency ( $\leq 10$ bp mismatch), leaving 32,991,450 sequences for contig assembly (Table 1). Using Ray v1.7 [22], 56,712 contigs were assembled and submitted to the MG-RAST pipeline [21]. Post quality control, 53,785 sequences (94.8\%), with a mean length of $160 \pm 55 \mathrm{bp}$, were used for further analysis (Table 1). When the contigs were analyzed using a best hit approach through MG-RAST, they aligned predominantly to the phyla of Proteobacteria (65.1\%) and Firmicutes (34.6\%, Figure 2). The contigs aligned to 194 known genomes at the genus level, predominantly Pseudomonas (61.1\%), Staphylococcus (33.4\%) and Streptococcus (0.5\%), with the highest level of diversity at the genus level within the Proteobacteria phylum (125 different genera, Figure 2). These results are similar to the best hit analysis performed with the non-assembled sequences in that the majority of sequences are from Staphylococcus and Pseudomonas, but differ in their proportion (Figure 1). Contigs matching viral genomes were observed $(<0.04 \%)$, including phages derived from Pseudomonas and Staphylococcus (Figure 2). Contigs also aligned to the genomes of humans, gorillas, chimps and orangutans, likely due to the $60 \%$ identity criteria used (Figure 2). The observation of some of the genera, including Staphylococcus, Pseudomonas and Pantoea, was further validated through the presence of their rRNA ORFs (Additional file 3).

\section{Open reading frames within human milk}

A total of 41,352 ORFs were predicted using MG-RAST, of which $82 \%$ were annotated (33,793 ORFs), and $18 \%$ were unrecognized (7,559 sequences, Table 1). A total of 30,128 ORFs corresponded to a functional category (Figure 3). For example, many ORFs encoded proteins for basic cellular function, including those for respiration (4.2\%), cell signaling (4.8\%), RNA (7.0\%), DNA (2.6\%), and amino acid metabolism (5.3\%, Figure 3$)$. ORFs encoding proteins for carbohydrate metabolism (5.7\% of all ORFs) included those for lactose metabolism (oligosaccharide, 


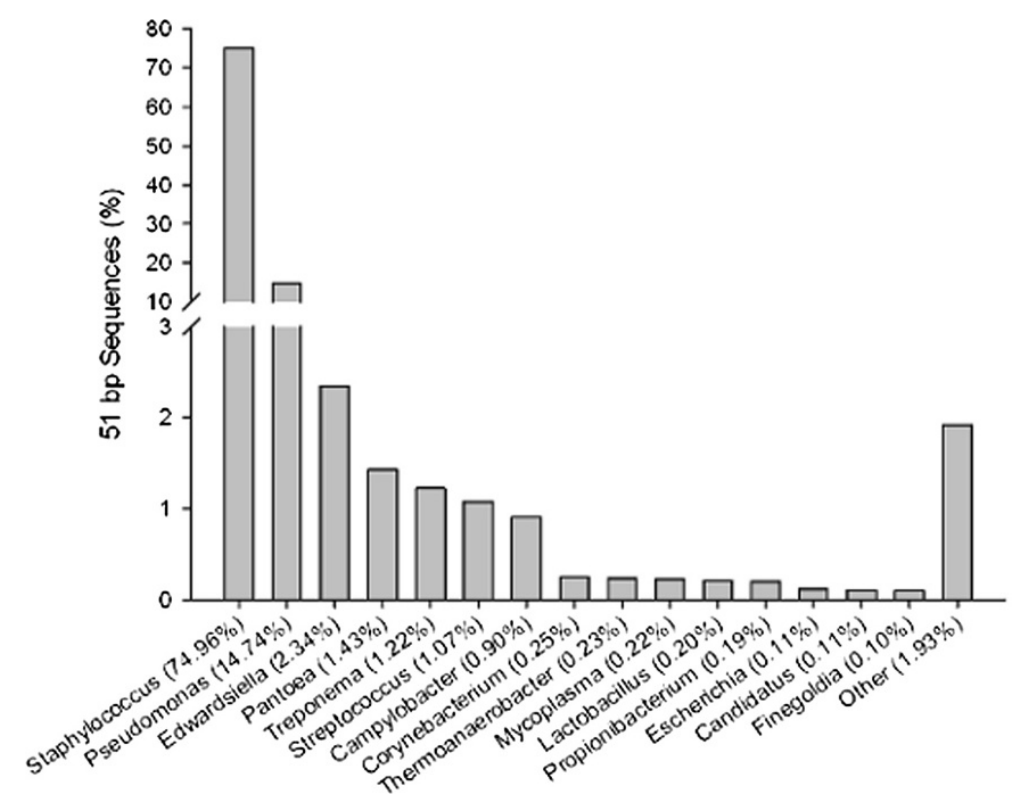

Figure 1 Best hit analysis of $\mathbf{5 1}$ bp DNA sequences from human milk. DNA from human milk was sequenced using Illumina sequencing followed by alignment to known prokaryotic genomes. Sequences $(75,521,216)$ were BLATed against 1,731 known prokaryotic genomes imported from NCBI (min 95\% identity), with 1,331,996 sequences aligning to 370 prokaryotic genera. Other refers to genera each representing $<0.1 \%$ of all sequences.

6.7\%), but none for human milk oligosaccharide metabolism (Figure 3), likely due to the lack of sequences aligning to the genome of Bifidobacteria (Figure 2). Virulencerelated ORFs (4.5\% of all ORFs) included those for antibiotic resistance (60.2\%), adhesion (17\%), bacteriocins (2.7\%), as well as others (Figure 3). Stress-related ORFs (4.0\% of all ORFs) included those for oxidative stress (40.3\%), osmotic stress $(20.2 \%)$, heat and cold shock $(12.0 \%$ and $4.0 \%$, respectively) and many others (Figure 3).

Table 1 Contig assembly and open reading frame (ORF) prediction of Illumina reads (51 bp) from human milk

\begin{tabular}{lr}
\hline Sequenced reads (51 bp) & $\mathbf{2 6 1 , 5 3 2 , 2 0 4}$ \\
Matching human & $186,010,988$ \\
Matching prokaryotic & $1,331,996$ \\
Used in contig assembly ${ }^{1}$ & $32,991,450$ \\
Contigs & $\mathbf{5 6 , 7 1 2}$ \\
Post quality control & 53,785 \\
Average length (bp) & $160 \pm 55$ \\
Total length (bp) & $8,630,997$ \\
Predicted ORFs & $\mathbf{4 1 , 3 5 2}$ \\
Annotated & 33,793 \\
rRNAs & 103 \\
Functional category & 30,128 \\
Unrecognized & 7,559 \\
\hline
\end{tabular}

${ }^{1}$ all sequences not matching the human genome ( $\leq 10 \mathrm{bp}$ mismatch).

\section{Human milk metagenome compared to mothers' and} infants' feces

The metagenome of human milk was compared to that of feces from 10 unrelated infants (five BF and five FF) and three unrelated mothers (Figure 4). Using a best hit analysis at the phylum level, contigs from human milk were dissimilar from contigs from feces in regards to the lack of diversity within the human milk metagenome, as over $99 \%$ of the contigs were from just two phyla, Proteobacteria and Firmicutes (65.1\% and 34.6\%, respectively, Figure 4). BF-infants' feces had a high proportion of Actinobacteria (70.4\%), followed by FF-infants' feces (27.3\%), mothers' feces $(12.6 \%)$, and human milk $(0.15 \%)$. The proportion of Proteobacteria in the human milk metagenome (65.1\%) was most similar to that of BF-infants' feces (10.8\%), but was significantly different from FF-infants' feces and mothers' feces $(7.5 \%$ and $4.3 \%$, respectively, $P<0.05$, Figure 2 and Additional file 4). The metagenomes of FFinfants' feces and mothers' feces were most similar in regards to their high proportion of Bacteroidetes $(17.6 \%$ and $20.6 \%$, respectively). Conversely, when using a lowest common ancestor approach at the phylum level in comparison to the best hit analysis, human milk appeared more similar to the fecal metagenomes in terms of an increase in diversity (Additional file 5), but was still dominated by Proteobacteria (38.5\%). Also, using the lowest common ancestor analysis increased the proportion of contigs aligning to Actinobacteria in human milk ( $0.15 \%$ to $11.58 \%)$, as well as in mothers' feces (12.6\% to $30.6 \%)$. 


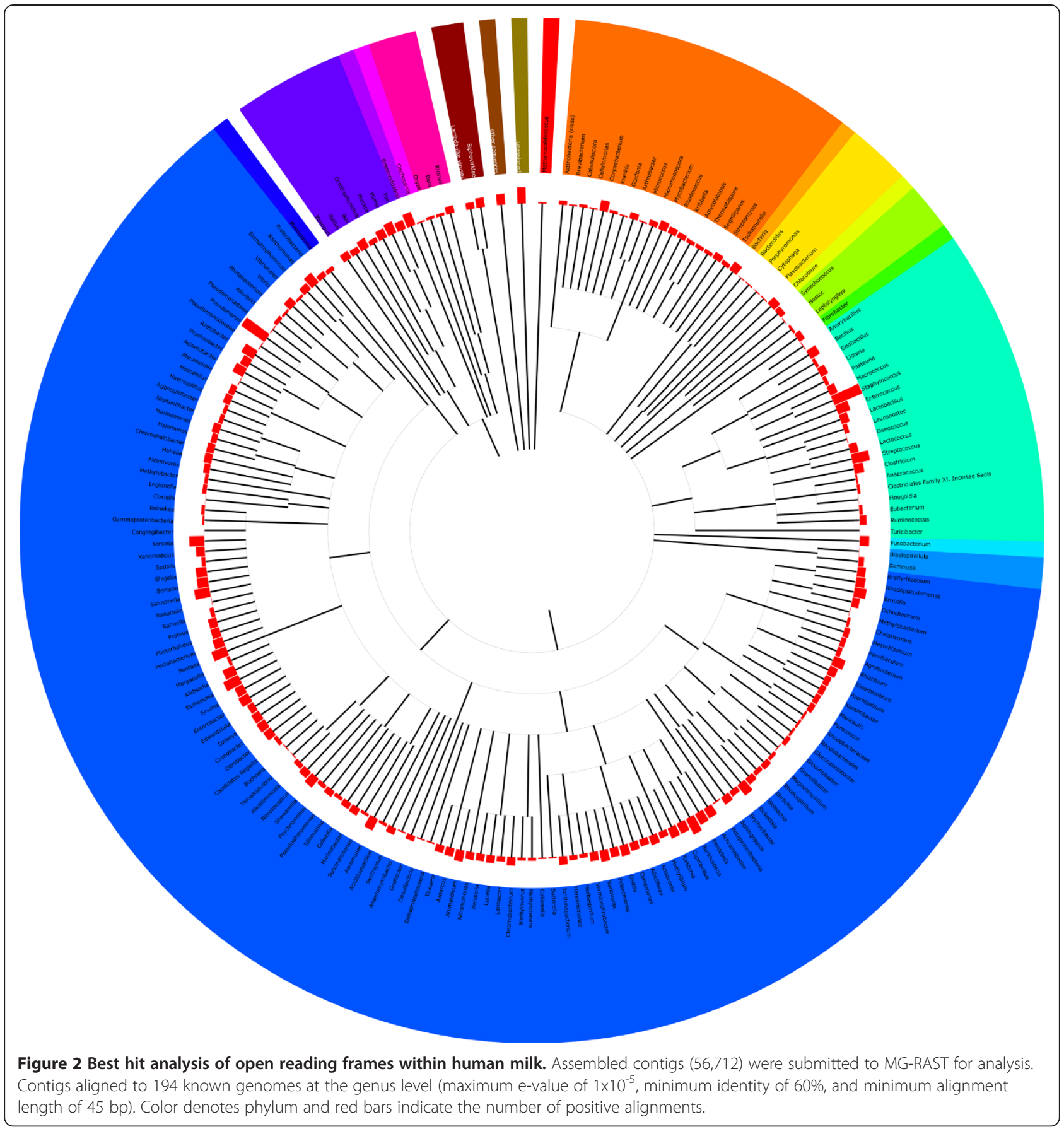

The metagenomes of human milk and feces were also compared at the functional level (Figure 5). The functional ORF profile of the human milk metagenome is similar to that of each fecal metagenome, but two fecal profiles were even more similar, for example BF-versus FF-infants' feces, as seen using pair-wise comparison plots (Figure 6). The human milk metagenome is most dissimilar from that of FF-infants' feces as 17 out of the 26 functional categories contain a significantly different proportion of the ORFs (Figure 6). The three fecal metagenomes had a significantly higher proportion of ORFs encoding genes for dormancy and sporulation (2.3\%, 2.3\% and 2.7\%, for BF-infants', FF-infants' and mothers' feces, respectively) than did the human milk metagenome (no associated ORFs, Figures 5 and 6). Both BF- and FF-infants' fecal metagenomes had significantly higher proportions of cell division $(3.5 \%$ each, respectively) and phosphorus metabolism related ORFs (3.1\% and $3.0 \%$, respectively) than did the human milk metagenome (2.3\% and $2.1 \%$, Figures 5 and 6$)$. The 


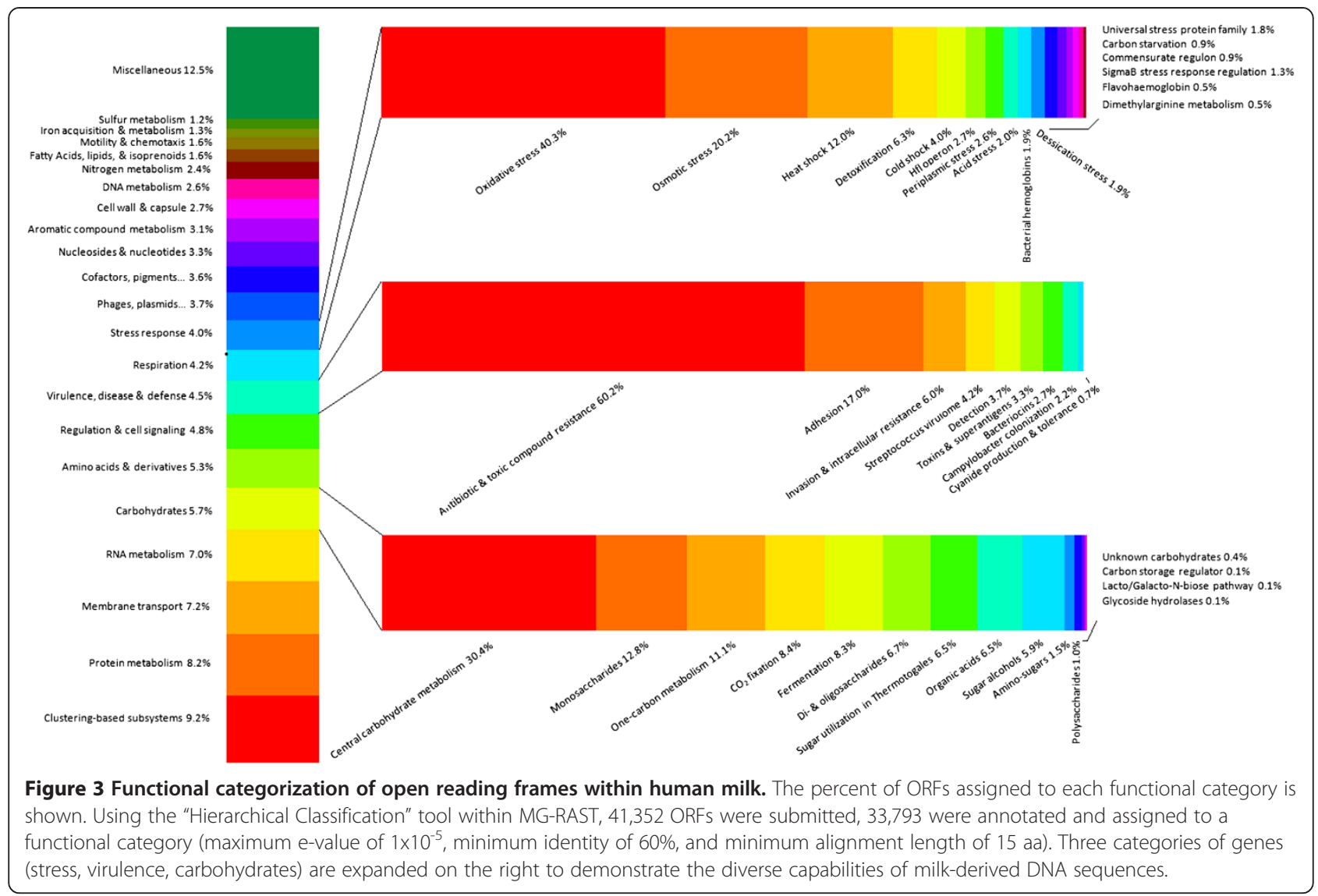

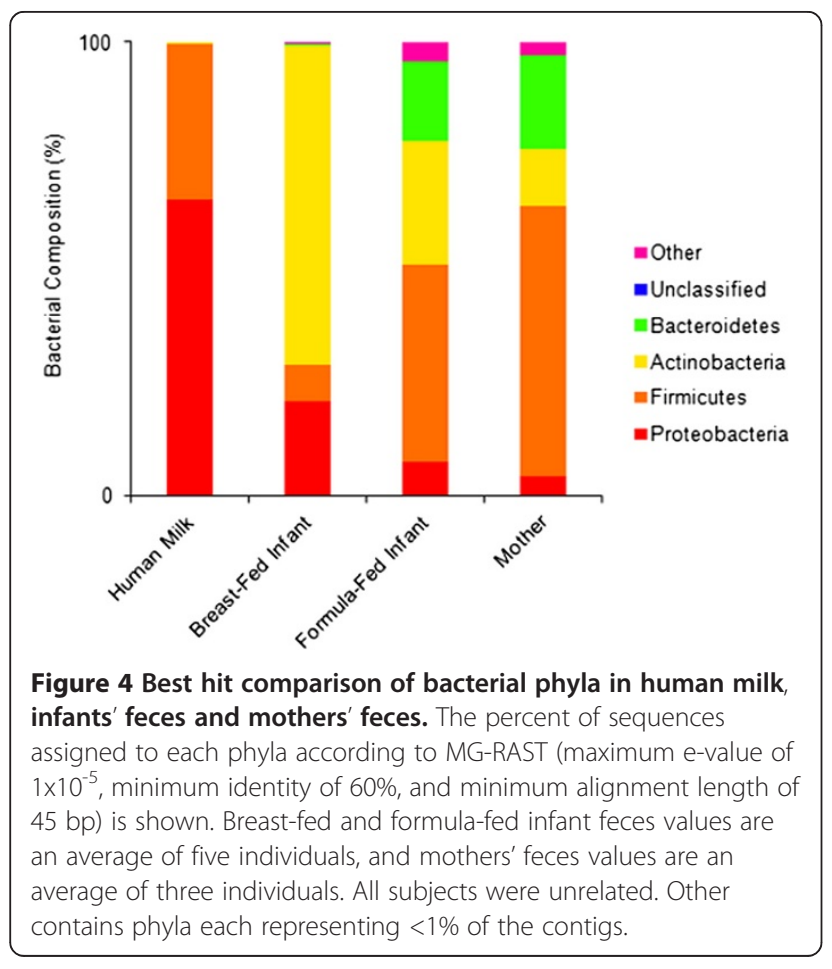

human milk metagenome, in comparison to BF- and FFinfants' feces, did, however, have significantly higher proportions of membrane transport (5.0\% compared to $4.0 \%$ and $4.0 \%$ ), nitrogen (3.5\% compared to $3.1 \%$ and $3.0 \%$ ) and RNA metabolism (4.9\% compared to $4.1 \%$ and $4.3 \%$ ), cell regulation (4.4\% compared to $3.5 \%$ and $3.3 \%$ ), respiration (4.3\% compared to $3.4 \%$ and $3.4 \%$ ), stress response (4.2\% compared to $3.7 \%$ and $3.5 \%$ ) and virulence-related ORFs (4.4\% compared to $3.7 \%$ and $3.7 \%$, Figures 5 and 6 ).

Immune-modulatory DNA motifs in human milk and feces When contigs were searched for the presence of immune suppressive motifs, TCAAGCTTGA was found in $0.02 \%$ of the human-milk assembled contigs (11 sites, Table 2) with an occurrence 1.5 times that of the human genome alone (once per $844,000 \mathrm{bp}$ compared to once per 1,276,500 bp in the human genome, Z-score -1.6). The contigs positive for TCAAGCTTGA aligned to the genomes of Pseudomonas (45\%), Nocardia (9\%), Staphylococcus (9\%) and contigs of unknown origin (36\%, Table 3). When the contigs from BF-infants' feces, FF-infants' feces and mothers' feces were scanned for TCAAGCTTGA, it was found at a relative occurrence of 1.19, 1.64, and 1.64 times that in the human genome, respectively (Table 2). Another immune suppressive site, 


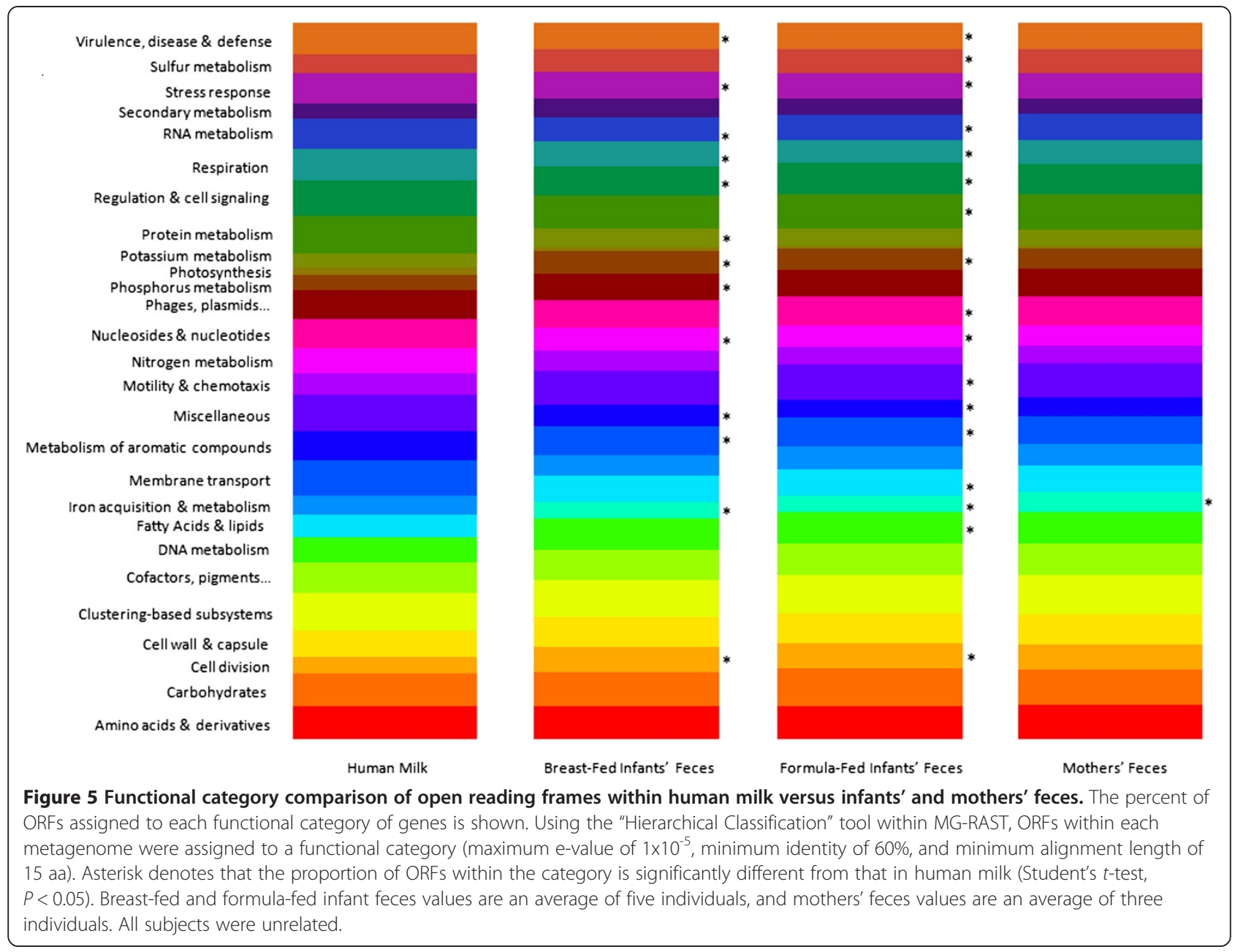

TTAGGG was observed 1,684 times in the human milk metagenome (3.2\% of contigs), and at a relative occurrence 0.48 times that of the human genome (once per 5,600 bp compared to once per 2,670 bp, Z-score 22.54, Table 2). Contigs containing TTAGGG corresponded to genomes of Staphylococcus (59\%), Pseudomonas (10\%), Lactobacillus (0.5\%), 21 other known prokaryotic genomes (2.7\%), and contigs from unknown genomes (27\%, Table 3). When the contigs from BF-infants' feces, FF-infants' feces and mothers' feces were scanned for TTAGGG, this sequence was observed at a relative occurrence of $0.33,0.18$ and 0.26 times that in the human genome, respectively (Table 2). Assembled contigs were also searched for the presence of synthetically-assembled immune suppressive or immune stimulatory DNA motifs ( 7 and 5 motifs, respectively), such as those used in vaccine production (Additional file 6 [23-27]). No synthetically-assembled sequences were observed in the human-milk contigs, whereas three motifs were found in less than $5 \times 10^{-4} \%$ of contigs from the fecal metagenomes (maximum of 4 hits per 834,774 contigs, Additional file 6).

\section{Discussion}

\section{Genera within human milk}

Determining the human milk metagenome, a bodily fluid notably absent from the human microbiome project [28], is crucial for enabling better insight on the process of infant GI colonization and immune development. By pooling DNA from ten human milk samples and subjecting it to Illumina sequencing we have demonstrated the large diversity of the human milk metagenome with over 56,000 contigs aligning to 177 bacterial genera (Figure 2). Previous studies investigating the microbiome of human milk have used both culture-dependent and independent approaches. Using 16S rRNA sequencing, Hunt et al. have reported several predominant species in human milk including a core of genera found in 15 human milk samples across time: Streptococcus, Staphylococcus, Serratia, Pseudomonas, Corynebacteria, Ralstonia, Propionibacteria, Sphingomonas, and Bradyrhizobiaceae [17]. Other studies showed colostrum was populated mostly by Weisella and Leuconostoc, followed by Staphylococcus, Streptococcus, and Lactococcus, and that Akkermansia were more prevalent in overweight mothers [20,29]. Using 

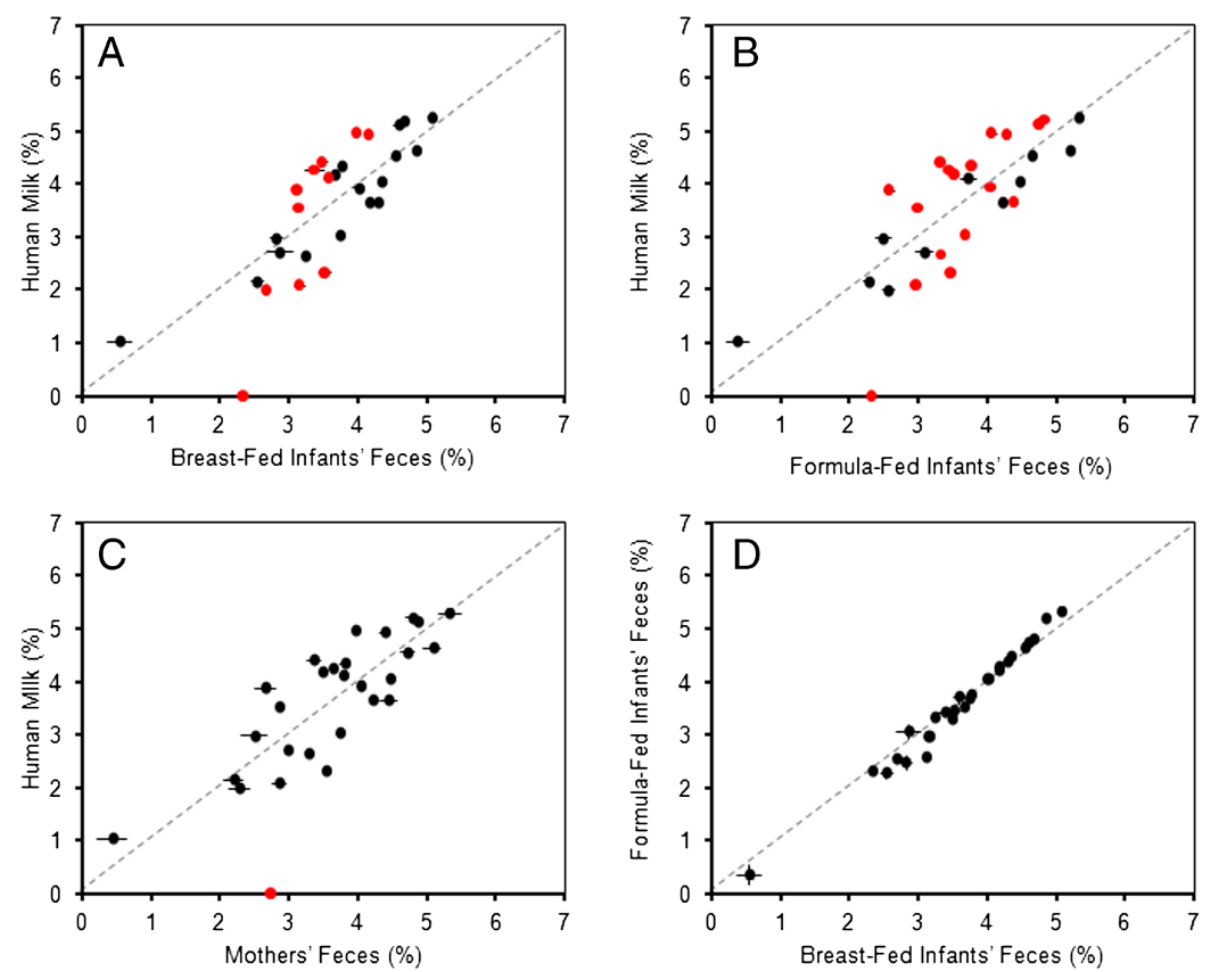

Figure 6 Pair-wise comparison of categorized open reading frames from human milk versus infants' and mothers' feces. Pair-wise comparisons for the human milk metagenome versus (A) breast-fed infants' feces, (B) formula-fed infants' feces and (C) mothers' feces are shown. For comparison, a plot of breast-fed infants' feces and formula-fed infants' feces (D) is also shown. Each point represents a different SEED subsystem and its relative abundance within the human milk metagenome compared to the fecal metagenomes. Points lying on or near the dotted line have equal or similar abundance in both metagenomes. Points closer to the $x$-axis are more abundant in the feces metagenome, whereas points closer to the $y$-axis are more abundant in the human milk metagenome. Red dots signify those with significantly different proportions between the two metagenomes (Student's $t$-test, $P<0.05$ ). Breast-fed and formula-fed infants' feces values are an average of five individuals, and mothers' feces values are an average of three individuals. All subjects are unrelated.

a best hit analysis of the $51 \mathrm{bp}$ Illumina reads, alignments for Akkermansia, Propionibacteria, Sphingomonas and Weisella were observed (Additional file 2), but because of the small number of base pairs used for the alignment (51 bp) and the lack of assembled contigs associated with these microbes, their presence in our milk samples is a tentative identification. Using PCR-denaturing gradient gel electrophoresis and quantitative PCR, two studies from Martin et al. reported the presence of Bifidobacterium breve, $B$. adolescentis, $B$. bifidum and $B$. dentium in human milk, which differs from our findings (Figure 2, $[15,16])$. This is likely due to the method of DNA

Table 2 Occurrence of immune suppressive motifs in various metagenomes

\begin{tabular}{lrrrr}
\hline Sequence & Number of hits & \multicolumn{2}{c}{ Base pairs per hit } & Relative occurrence $(\mathbf{Z}$-score) \\
\hline TCAAGCTTGA & 11 & 844,000 & (Human Milk) & $1.51(-1.6)$ \\
& 344 & $1,077,000$ & (BF Infant) & $1.19(-0.74)$ \\
& 124 & 779,000 & (FF Infant) & $1.64(-1.84)$ \\
TTAGGG & 268 & 777,000 & (Mother) & $1.64(-1.85)$ \\
& 2,245 & $1,276,500$ & (Human Genome) & $0.48(22.54)$ \\
& 1,684 & 5,600 & (Human Milk) & $0.33(42.54)$ \\
& 18,118 & 8,200 & (BF Infant) & $0.18(94.85)$ \\
& 16,410 & 15,000 & (FF Infant) & $0.26(57.92)$
\end{tabular}

DNA motifs TTAGGG and TCAAGCTGA were searched for in contigs derived from human milk, breast-fed infants' feces (BF infant), formula-fed infants' feces (FF infant) and mothers' feces. Relative occurrence is in comparison to the human genome. 
Table 3 Occurrence of immune suppressive motifs TTAGGG and TCAAGCTTGA in contigs from human milk

\begin{tabular}{llr}
\hline Sequence & Genus & Number of hits \\
\hline TCAAGCTTGA & Pseudomonas & 5 \\
& Nocardia & 1 \\
Staphylococcus & 1 \\
TTAGGG & Unknown & 4 \\
& Staphylococcus & 1000 \\
& Pseudomonas & 169 \\
& Lactobacillus & 8 \\
& Bacillus & 6 \\
& Streptococcus & 6 \\
& Streptomyces & 4 \\
& Tetragenococcus & 4 \\
& Other & 25 \\
& Unknown & 461 \\
\hline
\end{tabular}

extraction used in our study, as we did not incorporate bead-beating as a means to extract DNA from the hard to rupture Bifidobacterium [30]. The differences between the previously reported microbial communities and our analysis may also be due, in part, to the geographic location of the mothers, which has been shown to greatly impact the microbiome of individuals [31]. Furthermore, other differences between our characterization of the milk microbiome and those previously reported may be attributed to the means of milk collection. In comparison to previous studies where human milk was expressed from an aseptic breast [13-20], the current method determines the total microbiome (i.e. metagenome) ingested by the infant (from a non-sterilized breast), indicative of what an infant would receive from its mother during suckling.

Because our samples were collected from a nonsterilized breast, it could be hypothesized the human milk metagenome reported here would be similar to that of the skin microbiome. Although no reference database was freely available within MG-RAST for comparison, the metagenome of human milk is similar to previously reported skin profiles in that there is a large proportion of Staphylococcus, which is found in moist areas of skin. These moist areas, such as the antecubital fossa (inner fold of the elbow), also contain Betaproteobacteria, such as Burkholderia and Bordetella, which are present in the milk metagenome (Figure 2 [32,33]). The human milk metagenome is also similar to drier areas of the skin such as the plantar heel, which contains Gamaproteobacteria such as Pseudomonas [32]. The human milk metagenome is, however, more similar to fecal microbiomes (as described in 16S rRNA studies) due to the large proportion of Firmicutes bacteria within human milk, which is a very minor member of the skin microbiome (Figure 4, [32,33]). Also, the skin of adults tends to contain a high level of
Propionibacteria, which notably tends to colonize the skin of cesarean-section birthed babies, whereas this genus is minimally represented in our human milk sample using a best hit analysis of the 51 bp Illumina reads $(0.2 \%$, Additional file 2, $[34,35])$. This observation suggests that mother's milk may prove useful as a skin lotion, to rebalance the skin microbiome of $\mathrm{C}$-section babies.

\section{Phylogenetic differences between human milk and feces} Comparing the metagenome of human milk to that of publicly available infants' and mothers' fecal profiles provides insight as to how human milk may lead to proper colonization of the infant gut. When comparing the human milk metagenome to the infant fecal metagenome, there are numerous differences. For example, the metagenome of BF-infants' feces contains a high proportion of Actinobacteria (70.4\%, Figure 4), which correlates with previous studies demonstrating a high abundance of Bifidobacterium in the feces of BF-infants whereas FFinfants had a more varied microbiota $[6,31,36]$. Contigs from human milk, however, aligned mostly with Proteobacteria and Firmicutes $(65.1 \%$ and $34.6 \%$, respectively, Figure 4). At the phylum level, the present milk metagenome was less diverse than the fecal metagenomes as over $99 \%$ of the contigs were from just two phyla, Proteobacteria and Firmicutes (Figure 4). FF-infants' feces and mothers' feces were similar in that they both contained contigs aligning to the phylum Bacteroidetes (17.6\% and $20.6 \%$, respectively), whereas Bacteroidetes was a very minor component of BF-infants' feces and human milk $(0.3 \%$ and $0.02 \%$, respectively, Figure 4$)$. Also, the similar proportion of Firmicutes in human milk compared to mothers' feces $(34.6 \%$ and $59.6 \%$, respectively, Figure 4) correlates with the hypothesis that mothers' milk may be inoculated by immune cells carrying bacteria from the GI tract of the mother to her breast [37-39]. This may be a mechanism by which the human milk microbiome is shaped by the general health of the mother, including her weight [20].

\section{Functionality of the human milk metagenome}

Using Illumina sequencing of all DNA within milk samples permits the prediction of ORFs within assembled contigs and allows for determination of the functional capability of the milk metagenome. A total of 41,352 ORFs were predicted, including those for basic cell function, as well as those that may enable the bacteria to remain in human milk, such as ORFs for carbohydrate metabolism (5.7\% of ORFs, Figure 3). The predominant carbohydrate in human milk, lactose, is a potential carbon source for human milk bacteria, and therefore the presence of ORFs associated with its metabolism $(6.7 \%$ of carbohydrate-associated metabolism, Figure 3) is expected. Another carbon source for bacteria in human 
milk is human milk oligosaccharides (HMOs), which cannot be digested by the infant [40]. These oligosaccharides, which are heavily fucosylated and readily digested by Bifidobacteria, are thought to be responsible for the colonization of BF-infants with high levels of Bifidobacteria [41]. Due to a lack of contigs aligning to Bifidobacteria (Figure 2), no ORFs encoding genes for HMOs were observed (Figure 3). Recently, HMOs have also been correlated with increased abundance of Staphylococcus within human milk, regardless of their inability to utilize the human milk oligosaccharides as a carbon source [42]. The predominance of Staphylococcus-aligning contigs in our milk samples supports these findings (Figure 2). Furthermore, there was a significantly higher number of ORFs related to nitrogen metabolism within the human milk metagenome in comparison to BF- and FF-infants' feces (Figure 5, $P<$ 0.05). Because human milk contains $1.48-2.47 \mathrm{~g}$ of nitrogen per $100 \mathrm{~g}$ of milk, the bacteria within human milk may use it as a nutrient source in addition to lactose and HMOs [43].

Human milk contains an abundance of immune cells, antibodies and antimicrobial proteins (such as lactoferrin, CD14, alpha-lactalbumin, and lysozyme), and therefore the bacteria residing within human milk must harbor mechanisms to combat the milk-endogenous immune system [44-46]. For example, the metagenome of human milk includes ORFs for stress response and defense $(4.0 \%$ and $4.5 \%$ of all ORFs, respectively) including those for oxidative stress $(40.3 \%$ of stress-related ORFs) and toxic compound resistance $(60.2 \%$ of defense ORFs, Figure 3). The human milk metagenome also contains ORFs for both heat and cold shock ( $12 \%$ and $4 \%$ of stress-related ORFs, Figure 3), which may enable the bacteria to persist in milk post-breast pumping, refrigeration and reheating. This may be of particular importance as human milk banks gain more popularity over time. For example, as described in a recent review by Urbaniak et al., some milk banks deem pasteurization of breast milk unnecessary, while others have an upper limit of $10^{5}$ organisms per $\mathrm{ml}$ [47]. In unpasteurized banked milk and in-home stored milk, if some organisms are able to survive the storage and re-heating process better than others, the bacterial profile of human milk may change to favor better surviving (and not necessarily more beneficial) bacteria. Furthermore, ORFs encoding genes related to virulence and disease $(4.5 \%$ of all ORFs, Figure 3), are also observed in the human milk metagenome. These ORFs could allow some of the human milk microbes, such as Staphylococcus aureus, to cause mastitis in humans when the balance of human milk-antimicrobials to microbes is tilted towards microbial growth [48]. For example, some bacteria within human milk harbor antibiotic resistance genes $(60.2 \%$ of virulence associated ORFs) allowing them to proliferate regardless of the mother's potential antibiotic use, and some bacteria are able to produce bacteriocins $(2.7 \%$ of virulence associated ORFs, Figure 3), which could impact the growth of other, less virulent, microbes within the community.

\section{Immune-modulatory landscape of the human milk metagenome}

Because human milk contains a broad spectrum of microbes at the genus level (Figure 2), it likely contributes significantly towards effective colonization of the infant GI tract. In the case of banked human milk, which is Holder pasteurized $\left(65^{\circ} \mathrm{C}\right.$ for $\left.5-30 \mathrm{~min}\right)$, most bacteria are destroyed, but their proteins and DNA remain [49]. The presence of non-viable bacteria and bacterial DNA in human milk, which are indistinguishable from live bacteria using our approach of DNA isolation and sequencing, may be a way to prime the infant immune system and lead to tolerance of the trillions of bacteria that will inhabit the gut following birth. For example, the immune suppressive motifs, TTAGGG and TCAAGCTTGA [11], are present in $3.0 \%$ and $0.02 \%$ of the 56,950 human milk-contigs, respectively $(1,684$ sites, and 11 sites, Table 2$)$. The occurrence of the immune suppressive motifs is similar to that in the metagenomes of BF- and FF infants' feces, as well as mothers' feces. This suggests that having a diverse community of microbes may lead to a similar abundance of immune suppressive motifs, regardless of the genera present in the sample. Interestingly, the immune suppressive motif TTAGGG was found in higher abundance in the human genome than in bacterial contigs (one per $2,670 \mathrm{bp}$ in the human genome compared to one per $5,600 \mathrm{bp}$ in the bacterial contigs, Table 2). Colostrum and mature human milk contain between $5 \times 10^{8}$ to $4 \times 10^{9}$ leukocytes/L and between $1 \times 10^{8}$ to $4 \times 10^{9}$ leukocytes/L, respectively, which are mostly macrophages (55\%-60\%) and neutrophils (30\%-40\%), with natural killer cells representing up to $12 \%$ of the population [50,51]. This suggests that ingestion of the mothers' DNA, through ingestion of her immune cells and any free circulating DNA may also lead to proper immune development through a balance of concomitant exposure to immune stimulatory bacterial CpGs and immune suppressive DNA in the mothers' genome and bacterial genomes.

\section{Conclusions}

Current microbiome studies characterizing the microbial communities of various anatomical niches have revealed vast differences between healthy individuals [28]. These differences can often be attributed to the host's environment and diet. As demonstrated previously by preliminary $16 \mathrm{~S}$ rRNA sequencing, the human milk microbiome is similar to other areas of the body in that its 
composition is unique to each individual [17]. Milk has evolved as the first nutrient source for mammals ex utero, with a high level of inter-mother diversity as to the proportions of bacterial genera, immune proteins and nutrients within it [29]. Perhaps, it is the diversity and/or sequences of DNA within the milk metagenome that is beneficial for infants, as opposed to any one specific bacterial genus or species. Recent reviews on human milk outline the phylotypes of bacteria within human milk, but only speculate on the function of the human milk microbiome due to a lack of data on the functional capacity of the microbes within human milk $[47,52]$. Because of this, we sought to better understand the human milk metagenome on a functional level rather than a solely phylogenetic level. The discovery of the abundance of immune suppressive DNA motifs observed within bacterial and human DNA from human milk, as well as ORFs within the human milk metagenome that allow bacteria to persist in the biological fluid provides a first glance into the functionality of the milk metagenome. Further studies should include those determining the efficacy of milk DNA to modulate the immune system in the GI tract, and a more exhaustive look at the metagenome of human milk and how it relates to infant health outcomes.

\section{Note added in Proof}

During revision of the manuscript, Everard et al published a report suggesting Akkermansia, a human mucus colonizer, helps control diet-induced obesity. Everard et al, 2013, Proc Natl Acad Sci USA doi/10.1073/pnas.1219451110.

\section{Methods}

\section{Donors and sample collection}

Breastfeeding women $(n=10)$ were recruited from the Children's Hospital of Eastern Ontario (CHEO, Ottawa, Canada) in accordance with the Research Ethics Board of CHEO and the University of Ottawa Research Ethics Board (2007303-01H). Informed consent was given by all participants, all donors were healthy, and milk was donated between 9 and 30 days postpartum. Milk samples were collected by either manual or electric breast pump expression into a sterile milk collection bag (Medela AG, Baar, Switzerland). To better represent a milk sample that would be received by the infant, breasts were not sterilized prior to collection. Samples were immediately frozen and then stored at $-70^{\circ} \mathrm{C}$.

\section{DNA isolation}

Milk samples $(1 \mathrm{~mL})$ were centrifuged at 5,000 $\times g$ for 10 minutes to pellet eukaryotic cells. Prokaryotic cells were pelleted from milk serum by centrifugation at $13,000 \times g$ for 15 minutes. Pellets were resuspended in $2 \mathrm{~mL}$ phosphate buffered saline with $1 \%$ Triton X-100 and incubated for 2 hours at $37^{\circ} \mathrm{C}$ to lyse any remaining eukaryotic cells. Bacteria were pelleted by centrifugation at $13,000 \times g$ for 15 minutes and pellets were resuspended in $500 \mu \mathrm{L}$ TE with $30 \mu \mathrm{L}$ of $10 \%$ sodium dodecyl sulfate and $5 \mu \mathrm{g}$ proteinase K. Samples were incubated for 2 hours at $37^{\circ} \mathrm{C}$, and DNA was isolated using phenol/chloroform as previously described [53]. DNA pellets were resuspended in $50 \mu \mathrm{L}$ TE buffer and pooled. A total of $\sim 4 \mu \mathrm{g}$ of double stranded DNA was isolated as quantified with Quant-iT PicoGreen (Invitrogen, Burlington, ON, Canada) using a Typhoon Trio Imager and Image Quant TL software (GE Healthcare, Waukesha, WI, USA). DNA integrity was also determined by agarose gel electrophoresis prior to sequencing.

\section{DNA sequencing, filtering and contig assembly}

The pooled DNA sample was sequenced seven independent times by StemCore Laboratories (Ottawa, Ontario, Canada). DNA was prepared according to the DNA sample preparation protocol 1003806 Rev. B for Illumina sequencing (Illumina Inc, San Diego, CA, USA). Sequencing was performed using an Illumina GAIIx Genome Analyzer and Illumina CASAVA analysis pipeline (v 1.7.0). Sequences were aligned to the human genome (hg19/ NCBI37) with a stringency of 2 bp mismatching using ELAND (Illumina Inc). Prokaryotic genomes (1,731 genomes) were imported from NCBI. Sequences were aligned to the genomes using BLAT (Kent Informatics, Inc.) and sorted via best hit analysis to genera according to "List of Prokaryotic Names with Standing in Nomenclature" (http://www.bacterio.cict.fr/, accessed February 2012). Unidentified sequences were further filtered by using BLAT against the human genome with a stringency of $\leq 10$ mismatches or gaps. Both prokaryotic and remaining unknown sequences were assembled into contigs using Ray v1.7 [22].

\section{Contigs, ORF prediction and characterization}

Assembled contigs were uploaded to the MG-RAST pipeline [21]. Organism abundance was analyzed using a lowest common ancestor approach with a maximum evalue of $1 \times 10^{-5}$, a minimum identity of $60 \%$, and a minimum alignment length of 15 measured in amino acids for protein and base pairs for RNA databases. A functional abundance analysis of ORFs was performed using "Hierarchical Classification" by comparing to subsystems with a maximum e-value of $1 \times 10^{-5}$, a minimum identity of $60 \%$, and a minimum alignment length of 15 measured in amino acids for protein and base pairs for RNA databases. Previously reported and publicly available metagenomes of feces from five unrelated BFinfants, five FF-infants (metagenome IDs: USinfTW4.1, 6.1, 10.1, 11.1, 12.1, 13.1, 15.1, 19.1, 20.1, and 21.11) and three unrelated mothers (metagenome IDs: USchp $[1,3,4,18,33] \mathrm{mom})$ were compared at the phylum level to 
the human milk metagenome within MG-RAST using the same lowest common ancestor analysis described above [21]. The mean percent alignments of the individuals were used in Figure 4 and Additional files 4 and 5. The normalized mean percent of ORFs in each functional category was used in Figures 5 and 6. Metagenome comparisons were statistically compared by Student's $t$-tests $(P<0.05)$ using SigmaPlot (Systat Software, Inc., San Jose, CA, USA).

\section{Immune-modulatory motif identification}

An identity of $100 \%$ was used to search for immunemodulatory motifs by alignment with assembled contigs from the human milk metagenome (56,712 contigs) or the fecal metagenomes described above $(834,774,64,662$ and 553,391 contigs from BF-infants', FF-infants' and mothers' feces, respectively). The human genome $(2,865,822,365 \mathrm{bp})$ was used as a comparative reference. $Z$-score was calculated using the formula $Z=(O-E) /$ Stdev, where $\mathrm{O}$ was the observed number of hits and $\mathrm{E}$ was the expected number of hits using the formula $\mathrm{E}=$ $\left(L_{\text {cont }}\right)\left(\mathrm{N}_{h /} \mathrm{L}_{h}\right)$ where $L_{\text {cont }}$ was length of sequences or assembled contigs, $\mathrm{N}_{h}$ was number of sites found in the human genome (or compiled bacterial genomes); Stdev was the standard deviation of occurrence of each motif in $22+\mathrm{X}+\mathrm{Y}$ human chromosomes.

\section{Availability of supporting data}

The data set supporting the results of this article is available in the MG-RAST repository, under the project name Human_milk_microbiome, http://metagenomics. anl.gov/linkin.cgi?project $=2959$.

\section{Additional files}

Additional file 1: Abundance of DNA fragments in pooled human milk, sequenced seven times. This table contains the number of DNA sequences per run and their general alignments.

Additional file 2: Classification of 51 bp DNA sequences derived from human milk by best hit analysis. This table contains all genera with at least one alignment match to sequences from human milkderived DNA.

Additional file 3: Predicted open reading frames from human milk DNA sequences aligning to rRNA genes of known organisms. This table contains all rRNA genes and their corresponding genera found within the human milk metagenome.

Additional file 4: Pair-wise comparison of phyla abundance in human milk versus infants' and mothers' feces metagenomes. This graph demonstrates the similarities between the human milk metagenome and the fecal metagenomes.

Additional file 5: Lowest common ancestor comparison of bacterial phyla in human milk, and in infants' and mothers' feces. This figure shows the relative abundance of each phylum in the human milk metagenome as compared to the fecal metagenomes.

Additional file 6: Immune-modulatory DNA motifs sought in DNA sequences derived from human milk or feces. This table shows all synthetically-assembled DNA motifs and their references that were searched for within the human milk and fecal metagenomes.

\section{Abbreviations}

BF: Breast-fed; FF: Formula-fed; Gl: Gastrointestinal; CpG: Cytosine phosphate guanine; rRNA: Ribosomal RNA; ORF: Open reading frame; HMO: Human milk oligosaccharide; COG: Cluster of orthologous groups.

Competing interests

The authors declare they have no competing interests.

\section{Authors' contributions}

TLW designed the data analysis approach, interpreted results and wrote the manuscript. SH performed the data analysis for Figure 1, Tables 1, 2, 3 and Additional file 1: Table S1, Additional file 2: Table S2 and Additional file 6: Table S4. IA conceived and supervised the study and edited the paper. II supervised the bioinformatics analyses and edited the paper. All authors have read and approved the manuscript.

\section{Acknowledgements}

This work was funded by the Canadian Institutes of Health Research, Institute of Nutrition, Metabolism and Diabetes (grant 82826 to IA) and Canada Foundation for Innovation, Leaders Opportunity Fund/Ontario Research Fund (grant 22880 to II). TLW is supported by a Natural Sciences and Engineering Research Council (NSERC) Canadian Graduate Scholarship. We are grateful to Lynne Cullen and Dr. JoAnn Harrold of the Children's Hospital of Eastern Ontario for donor recruitment and milk collection. We would also like to thank Dr. Will Spencer of BMI for isolating DNA from human milk, Kathy Sheikheleslamy of StemCore Laboratories (Ottawa Hospital Research Institute, Ottawa, Canada) for her sequencing efforts, and Chris Porter and Gareth Palidwor for filtering Illumina outputs.

Received: 21 February 2013 Accepted: 10 May 2013

Published: 25 May 2013

\section{References}

1. Kramer MS, Guo T, Platt RW, Sevkovskaya Z, Dzikovich I, Collet JP, Shapiro S, Chalmers B, Hodnett E, Vanilovich I, Mezen I, Ducruet T, Shishko G, Bogdanovich N: Infant growth and health outcomes associated with 3 compared with 6 mo of exclusive breastfeeding. Am J Clin Nutr 2003, 78:291-295.

2. Ladomenou F, Moschandreas J, Kafatos A, Tselentis Y, Galanakis E: Protective effect of exclusive breastfeeding against infections during infancy: a prospective study. Arch Dis Child 2010, 95:1004-1008.

3. Meinzen-Derr J, Poindexter B, Wrage L, Morrow AL, Stoll B, Donovan EF: Role of human milk in extremely low birth weight infants' risk of necrotizing enterocolitis or death. J Perinatol 2009, 29:57-62.

4. Sangild PT, Siggers RH, Schmidt M, Elnif J, Bjornvad CR, Thymann T, Grondahl ML. Hansen AK, Jensen SK, Boye M, Moelbak L, Buddington RK, Westrom BR, Holst JJ, Burrin DG: Diet- and colonization-dependent intestinal dysfunction predisposes to necrotizing enterocolitis in preterm pigs. Gastroenterol 2006, 130:1776-1792.

5. Sodhi C, Richardson W, Gribar S, Hackam DJ: The development of animal models for the study of necrotizing enterocolitis. Dis Model Mech 2008, 1:94-98.

6. Harmsen HJ, Wildeboer-Veloo AC, Raangs GC, Wagendorp AA, Klijn N, Bindels JG, Welling GW: Analysis of intestinal flora development in breastfed and formula-fed infants by using molecular identification and detection methods. J Pediatr Gastroenterol Nutr 2000, 30:61-67.

7. Sakata S, Tonooka T, Ishizeki S, Takada M, Sakamoto M, Fukuyama M, Benno $Y$ : Culture-independent analysis of fecal microbiota in infants, with special reference to Bifidobacterium species. FEMS Microbiol Lett 2005, 243:417-423.

8. Clemente JC, Ursell LK, Parfrey LW, Knight R: The impact of the gut microbiota on human health: an integrative view. Cell 2012, 148:1258-1270.

9. Dalpke A, Frank J, Peter M, Heeg K: Activation of toll-like receptor 9 by DNA from different bacterial species. Infect Immun 2006, 74:940-946.

10. Gursel I, Gursel M, Yamada H, Ishii KJ, Takeshita F, Klinman DM: Repetitive elements in mammalian telomeres suppress bacterial DNA-induced immune activation. J Immunol 2003, 171:1393-1400.

11. Bouladoux N, Hall JA, Grainger JR, dos Santos LM, Kann MG, Nagarajan V Verthelyi $D$, Belkaid $Y$ : Regulatory role of suppressive motifs from commensal DNA. Mucosal Immunol 2012, 5:623-634. 
12. Lin PW, Nasr TR, Stoll BJ: Necrotizing enterocolitis: recent scientific advances in pathophysiology and prevention. Semin Perinatol 2008, 32:70-82.

13. Heikkila MP, Saris PEJ: Inhibition of Staphylococcus aureus by the commensal bacteria of human milk. J Appl Microbiol 2003, 95:471-478

14. Martin R, Heilig HG, Zoetendal EG, Jimenez E, Fernandez L, Smidt H, Rodriguez JM: Cultivation-independent assessment of the bacterial diversity of breast milk among healthy women. Res Microbiol 2007, 158:31-37.

15. Martin R, Jimenez E, Heilig H, Fernandez L, Marin ML, Zoetendal EG, Rodriguez JM: Isolation of bifidobacteria from breast milk and assessment of the bifidobacterial population by PCR-denaturing gradient gel electrophoresis and quantitative real-time PCR. App/ Environ Microbiol 2009, 75:965-969.

16. Collado MC, Delgado S, Maldonado A, Rodriguez J: Assessment of the bacterial diversity of breast milk of healthy women by quantitative realtime PCR. Lett App/ Microbiol 2009, 48:523-528.

17. Hunt KM, Foster JA, Forney LJ, Schutte UM, Beck DL, Abdo Z, Fox LK, Williams JE, McGuire MK, McGuire MA: Characterization of the diversity and temporal stability of bacterial communities in human milk. PLoS One 2011, 6:e21313.

18. Martin V, Manes-Lazaro R, Rodriguez JM, Maldonado-Barragan A: Streptococcus lactarius sp. nov., isolated from breast milk of healthy women. Int J Syst Evol Microbiol 2011, 61:1048-1052.

19. Martin V, Maldonado-Barragan A, Moles L, Rodriquez-Banos M, Campo RD, Fernandez $L$, Rodriguez JM, Jimenez E: Sharing of bacterial strains between breast milk and infant feces. J Hum Lact 2012, 28:36-44.

20. Cabrera-Rubio R, Collado MC, Laitinen K, Salminen S, Isolauri E, Mira A: The human milk microbiome changes over lactation and is shaped by maternal weight and mode of delivery. Am J Clin Nutr 2012, 96:544-551.

21. Meyer F, Paarmann D, D'Souza M, Olson R, Glass EM, Kubal M, Paczian T, Rodriguez A, Stevens R, Wilke A, Wilkening J, Edwards RA: The metagenomics RAST server - a public resource for the automatic phylogenetic and functional analysis of metagenomes. BMC Bioinformatics 2008, 9:386.

22. Boisvert S, Laviolette F, Corbeil J: Ray: simultaneous assembly of reads from a mix of high-throughput sequencing technologies. J Comput Biol 2010, 17:1519-1533.

23. Hartmann G, Krieg AM: Mechanism and function of a newly identified CpG DNA motif in human primary B cells. J Immunol 2000, 164:944-953.

24. Stunz LL, Lenert P, Peckham D, Yi AK, Haxhinasto S, Chang M, Krieg AM, Ashman RF: Inhibitory oligonucleotides specifically block effects of stimulatory $\mathrm{CpG}$ oligonucleotides in B cells. Eur J Immunol 2002, 32:1212-1222.

25. Peter M, Bode K, Lipford GB, Eberle F, Heeg K, Dalpke AH: Characterization of suppressive oligodeoxynucleotides that inhibit Toll-like receptor-9 -mediated activation of innate immunity. Immunology 2008, 123:118-128.

26. Ashman RF, Goeken JA, Latz E, Lenert P: Optimal oligonucleotide sequences for TLR9 inhibitory activity in human cells: lack of correlation with TLR9 binding. Int Immunol 2011, 23:203-214.

27. Zhang X, Gao M, Ha T, Kalbfleisch JH, Williams DL, Li C, Kao RL: The toll-like receptor 9 agonist, CpG-oligodeoxynucleotide 1826, ameliorates cardiac dysfunction after trauma-hemorrhage. Shock 2012, 38:146-152.

28. Huttenhower C, Gevers D, Knight R, Abubucker A, Badger JH, Chinwalla AT, Creasy HH, Earl AM, FitzGerald MG, Fulton RS, Giglio MG, Hallworth-Pepin K: Structure, function and diversity of the healthy human microbiome. Nature 2012, 486:207-214.

29. Collado MC, Laitinen K, Salminen S, Isolauri E: Maternal weight and excessive weight gain during pregnancy modify the immunomodulatory potential of breast milk. Pediatr Res 2012, 72:77-85.

30. de Boer R, Peters R, Gierveld S, Schuurman T, Kooistra-Smid M, Savelkoul P: Improved detection of microbial DNA after bead-beating before DNA isolation. J Microbiol Methods 2010, 80:209-211.

31. Yatsunenko T, Rey FE, Manary MJ, Trehan I, Dominguez-Bello MG, Contreras M, Magris M, Hidalgo G, Baldassano RN, Anokhin AP, Heath AC, Warner B, Reeder J, Kuczynski J, Caporaso JG, Lozupone CA, Lauber C, Clemente JC, Knights D, Knight $\mathrm{R}$, Gordon J: Human gut microbiome viewed across age and geography. Nature 2012, 486:222-227.

32. Grice EA, Kong HH, Conlan S, Deming CB, Davis J, Young AC, Bouffard GG, Blakesley RW, Murray PR, Green ED, Turner ML, Segre JA: Topographical and temporal diversity of the human skin microbiome. Science 2009 324:1190-1192.
33. Costello EK, Lauber CL, Hamady M, Fierer N, Gordon Jl, Knight R: Bacterial community variation in human body habitats across space and time. Science 2009, 326:1694-1697

34. Oh J, Conlan S, Polley EC, Segre JA, Kong HH: Shifts in human skin and nares microbiota of healthy children and adults. Genome Med 2012, 4:77

35. Dominguez-Bello MG, Costello EK, Contreras M, Magris M, Hidalgo G, Fiere $\mathrm{N}$, Knight R: Delivery mode shapes the acquisition and structure of the initial microbiota across multiple body habitats in newborns. Proc Natl Acad Sci U S A 2010, 107:11971-11975.

36. Wharton BA, Balmer SE, Scott PH: Sorrento studies of diet and fecal flora in the newborn. Acta Paediatr Jpn 1994, 36:579-584

37. Perez PF, Dore J, Leclerc M, Levenez F, Benyacoub J, Serrant P, SeguraRoggero I, Schiffrin EJ, Donnet-Hughes A: Bacterial imprinting of the neonatal immune system: lessons from maternal cells? Pediatrics 2007, 119:e724-732

38. Donnet-Hughes A, Perez PF, Dore J, Leclerc M, Levenez F, Benyacoub J, Serrant P, Segura-Roggero I, Schiffrin EJ: Potential role of the intestinal microbiota of the mother in neonatal immune education. Proc Nutr Soc 2010, 69:407-415.

39. Rescigno M, Rotta G, Valzasina B, Ricciardi-Castagnoli P: Dendritic cells shuttle microbes across gut epithelial monolayers. Immunobiology 2001 204:572-581.

40. Engfer MB, Stahl B, Finke B, Sawatzki G, Daniel H: Human milk oligosaccharides are resistant to enzymatic hydrolysis in the upper gastrointestinal tract. Am J Clin Nutr 2000, 71:1589-1596.

41. Zivkovic AM, German JB, Lebrilla CB, Mills DA: Human milk glycobiome and its impact on the infant gastrointestinal microbiota. Proc Natl Acad Sci U S A 2011, 108(Suppl 1):4653-4658.

42. Hunt KM, Preuss J, Nissan C, Davlin CA, Williams JE, Shafii B, Richardson AD, McGuire MK, Bode L, McGuire MA: Human milk oligosaccharides promote the growth of Staphylococci. Appl Environ Microbiol 2012, 78:4763-4770.

43. Corvaglia L, Battistini B, Paoletti V, Aceti A, Capretti MG, Faldella G: Nearinfrared reflectance analysis to evaluate the nitrogen and fat content of human milk in neonatal intensive care units. Arch Dis Child Fetal Neonatal Ed 2008, 93:F372-375.

44. Blais DR, Harrold J, Altosaar I: Killing the messenger in the nick of time: persistence of breast milk sCD14 in the neonatal gastrointestinal tract. Pediatr Res 2006, 59:371-376.

45. Lepage $P$, Van de Perre $P$ : The immune system of breast milk: antimicrobial and anti-inflammatory properties. Adv Exp Med Biol 2012, 743:121-137.

46. Spencer WJ, Binette A, Ward TL, Davis L, Blais DR, Harrold J, Mack DR, Altosaar I: Alpha-lactalbumin in human milk alters the proteolytic degradation of soluble CD14 by forming a complex. Pediatr Res 2010, 68:490-493.

47. Urbaniak C, Burton JP, Reid G: Breast, milk and microbes: a complex relationship that does not end with lactation. Womens Health (Lond Engl) 2012, 8:385-398.

48. Delgado S, Garcia P, Fernandez L, Jimenez E, Rodriguez-Banos M, de Campo R, Rodriguez JM: Characterization of Staphylococcus aureus strains involved in human and bovine mastitis. FEMS Immunol Med Microbiol 2011, 62:225-235

49. Espinosa-Martos I, Montilla A, Segura AG, Escuder D, Bustos G, Pallas C, Rodriguez JM, Corzo N, Fernandez L: Bacteriological, biochemical and immunological modifications in human colostrum after Holder pasteurisation. J Pediatr Gastroenterol Nutr 2013, 56(5):560-568. doi:10.1097/ MPG.0b013e31828393ed.

50. Goldman AS: The immune system of human milk: antimicrobial, antiinflammatory and immunomodulating properties. Pediatr Infect Dis $J$ 1993, 12:664-671.

51. Jin YY, Wei Z, Cao RM, Xi W, Wu SM, Chen TX: Characterization of immunocompetent cells in human milk of Han Chinese. J Hum Lact 2011, 27:155-162.

52. Jeurink PV, van Bergenhenegouwen J, Jimenez E, Knippels LM, Fernandez L, Garssen J, Knol J, Rodriguez JM, Martin R: Human milk: a source of more life than we imagine. Benef Microbes 2013, 4:17-30.

53. Wilson K: Preparation of genomic DNA from bacteria. Curr Protoc Mol Biol 2001, 00:2.4.1-2.4.5.

\section{doi:10.1186/1471-2180-13-116}

Cite this article as: Ward et al: Human milk metagenome: a functional capacity analysis. BMC Microbiology 2013 13:116. 